\title{
Nivel de Conocimiento y Adopción de Conductas de Riesgo para las Infecciones de Transmisión Sexual/VIH, en Estudiantes de la UNJBG de Tacna Año 2009
}

\author{
Level of Knowledge and Adoption of Risk Behavior for \\ Sexually Transmitted Infections/HIV, in Students at the UNJBG \\ in Tacna Year 2009
}

'Leandra Llanca Ramos. 2 Gladys Concori Cori

\section{RESUMEN:}

La presente investigación permitió determinar el nivel de conocimiento y la adopción de conductas de riesgo para contraer una Infección de Transmisión SexuallVIH, en los estudiantes de la Universidad Nacional Jorge Basadre Grohmann de Tacna. Para tal efecto se encuestaron a 150 estudiantes universitarios seleccionados al azar; correspondiendo el $50 \%$ a mujeres y $50 \%$ a varones. Los resultados nos indican que el nivel de conocimientos de los estudiantes universitarios varones es bueno en un $13.3 \%$, regular en un 76.0\%, y malo $10.7 \%$ y el de las estudiantes universitarias, es bueno $17.3 \%$, regular $42.7 \%$. y malo $70 \%$, por lo tanto se puede apreciar que existe un alto desconocimiento sobre aspectos sexuales para asumir comportamientos que conduzcan a la prácticade un sexo seguro.

Palahras clave: factores de riesgo, infecciones de transmisión sexual. conductas de riesgo, nivel de conocimientos.

\section{ABSTRACT:}

The present investigation allowed to determine the level of knowledge and the adoption of hazardous hehavior to acquire an sexually Iransmitted infection THI in National University Jorge Basadre Grohmann students in Tacha. For this purpose 150 university students were surveyed selected randomly, corresponding $1050 \%$ women and $50 \%$ male. Results show us that the level of knowledge of male university students is good with a $13.3 \%$, moderate with a $76.0 \%$, and had with a $10.7 \%$. Regarding the level of knowledge of female university student, is good with a 17.3\%, moderate with a $73.8 \%$ and bad with a $40 \%$, therefore it can be seen that there is a great amount of ignorance about sexual aspects to assume behaviors leading to a safer sex practice.

Keywords: risk factors, sexually transmitted infection. hazardous conducts. level of knowledge sex

\footnotetext{
1 Magister en Docencia Universitaria y (iestion Educativa, Licenciada en Obstetricia. Facultad de Ciencias de la Salud Universidad Nacional Jorge Basadre Grohmann

Master of Science con mención en Tecnologia Edacalis a, Licenciada en Obstetricia.

Facultad de Ciencias de la Salud. Unisersidad Nacional Jorge Basadre Grohmann
} 


\section{INTRODUCCIÓN}

Las infecciones de transmisión sexual (ITS) se encuentran entre las principales causas de enfermedad del mundo, con consecuencias económicas, sociales y sanitarias de gran repercusión en muchos paises. La aparición y la diseminación del virus de inmunodeficiencia humana (VIH) y del sindrome de inmunodeficiencia adquirida (SIDA) han tenido un impacto significativo sobre el manejo y control de las ITS. Simultáneamente, ha aumentado la resistencia de varios agentes patógenos transmitidos sexualmente a los agentes antimicrobianos, agravando los problemas terapéuticos.

Según Datos presentados por el Ministerio de Salud, tenemos que la presencia de ITS/VIH,va incrementándose, pese a conocer las vías de contagio existentes, conllevando ello a que se pueda encontrar también el contagio a través de la via vertical, MadreNiño, esta última se pensaba que no se produciría, sin embargo ya se tiene reportes que ésta va aumentando. generando un incremento en las tasas de morbimortalidad materna y neonatal, considerándose hoy en día un problema en la Salud Pública.

Por ello interesa conocer las conductas de riesgo que adoptan los jóvenes estudiantes universitarios, pues se ha observado que hay un incremento permanente de las Infecciones de transmisión sexual. predominando el mismo en los jóvenes menores de 25 años; este fenómeno se atribuye a los siguientes factores:

- Inicio más temprano de las relaciones sexuales

- Menor temor a las ITS por la existencia de nuevos antibióticos

- Mayor inmigración y emigración de la población.

- Preferencia en el uso de métodos anticonceptivos hormonales y D.I.U. en detrimento de los métodos de barrera.

- Falta de medidas de prevención contra las ITS en especial en los paises en vías de desarrollo.

\section{- Falta de Educación Sexual}

Un alto porcentaje de personas infectadas con el VIH, afirman infectarse por vía sexual. La edad de contagio oscila entre los 15 y 23 años (MINSA, 2002)

Los adolescentes en el Perú constituyen un $22.3 \%$ de la población total, de los cuales un $10 \%$ vive en condiciones de extrema pobreza, lo cual a su vez supone falta de oportunidades y una pobre o deficiente comunicación. (Pereyra, 2002).

Si antes un $8 \%$ de los casos de infección de VIH involucraba a adolescentes, ahora constituye un $15 \%$, formando parte de un grupo muy vulnerable con un alto riesgo de contagio.
La mayor tasa de incidencia del SIDA en el Perú, se encuentra en los departamentos de Lima, Callao, Loreto, La Libertad, Piura, Arequipa, Junín, Lambayeque, Tumbes y Tacna.

La población más vulnerable se encuentra en los departamentos de la Costa y Selva, en ciudades densamente pobladas, estratos muy pobres y con un alto porcentaje de gente joven.

Las infecciones de transmisión sexuales (ITS), son un grupo de enfermedades que se adquieren fundamentalmente por contacto sexual, afectan a personas de cualquier edad, aunque son los adolescentes y los jóvenes, los grupos poblacionales más afectados. Se presentan más frecuentes en aquellos individuos que mantienen conductas $y$ actitudes sexuales riesgosas (como tener más de una pareja sexual, practicar sexo en forma no segura, no usar condón profiláctico, etc). La gente joven es más vulnerable a las ITS, fundamentalmente por razones de comportamiento. En esta etapa se desarrollan además, emociones concomitantes con la maduración sexual genital, por el mayor interés que existe en el sexo opuesto y en su mismo sexo.

En la actualidad, a pesar de los notables adelantos en los conocimientos médicos y el desarrollo de la atención primaria en nuestro medio, ha habido un incremento de las ITS.

La iniciación sexual se presenta en los adolescentes a edades cada vez más tempranas, lo que conlleva a un mayor número de parejas sexuales y por ende un incremento en el riesgo de contraer una ITS.

\section{MATERIALESY MÉTODOS}

El presente estudio es de carácter descriptivo, prospectivo y de corte transversal. La muestra estuvo constituida por los estudiantes universitarios de la Universidad Nacional Jorge Basadre Grohmann de Tacna, de los cuales se seleccionaron al azar una muestra constituida por 150 estudiantes, correspondiendo el $50 \%$ a mujeres y $50 \%$ a varones.

La recolección de los datos se realizó a través de la aplicación de una encuesta y para analizar los datos obtenidos se utilizó estadistica no paramétrica Prueba de Chi cuadrado y Rho de Spearman para el análisis bivariado

\section{RESULTADOS}

Las edades de los estudiantes universitarios varones oscilan de 16-19 años en un 34.7\%, de 20-22 años el $29.3 \%$, de 23 - 25 años el $22.7 \%$ y más de 25 años el $13.3 \%$. Las estudiantes universitarios mujeres oscilan de $16-19$ años en un $49.3 \%$, de 20-22 años el $22.7 \%$, de 23 - 25 años el $20 \%$ y más de 25 años el $8 \%$. Es decir aproximadamente el $80 \%$ entre hombres y mujeres se encuentran entre las edades de 16 a 19 años es decir en etapa de adolescencia. 
Los estudiantes universitarios varones son solteros en un $88 \%$, casado $4 \%$, conviviente $8 \%$. Las estudiantes universitarios mujeres son solteras en un $90.7 \%$, casadas $6.7 \%$, convivientes $2.6 \%$, es decir el $90 \%$ de estudiantes en general fueron solteros.

Los estudiantes universitarios tuvieron su primera relación sexual entre los 16-20 años en un $65.3 \%$ y 21 25 años en un $4.1 \%$. Mientras que las estudiantes universitarias tuvieron su primera relación sexual entre los 16-20 años en un $66 \%$ y $21-25$ años en un $10.7 \%$. Dicho resultado es similar a otros estudios realizados en población de adolescentes y jóvenes al señalar que las edades de inicio de las primeras relaciones coitales se encuentra entre los 16 y 20 años en los varones. mientras que las mujeres muestran un inicio más tardio entre los 21 a 25 años, los cual se podría explicar porque la mujer muestra conductas orientadas a mayor responsabilidad respecto de su sexualidad y frente a las ITS y VIH/SIDA.

Por otra parte se encontró que sólo el $16.2 \%$ de varones señalo mantener relaciones coitales con más de una persona y las mujeres lo niegan. La práctica sexual más frecuente en los varones es el sexo oral-vaginal en un $72.1 \%$, vaginal-oral-anal $23.5 \%$, anal-vaginal $2.9 \%$ y el vaginal $1.5 \%$, en cambio en las mujeres la más frecuente fue el sexo vaginal en un $69.2 \%$ y el sexo oralvaginal en un $30.8 \%$.

En los estudiantes universitarios varones el nivel de conocimiento es bueno $13.3 \%$, regular $76.0 \%$, y malo $10.7 \%$ en cambio en las mujeres el nivel de conocimiento es bueno $17.3 \%$, regular $42.7 \%$, y malo en un $40 \%$.

Se pudo establecer que los estudiantes varones presentaron una actitud muy desfavorable en un $10.3 \%$, desfavorable $61.8 \%$, favorable $23.5 \%$ y muy favorable $4.4 \%$ en cambio en las estudiantes mujeres, el nivel de actitud fue muy desfavorable ninguno, desfavorable $30.8 \%$, favorable $69.2 \%$ y muy favorable ninguno.

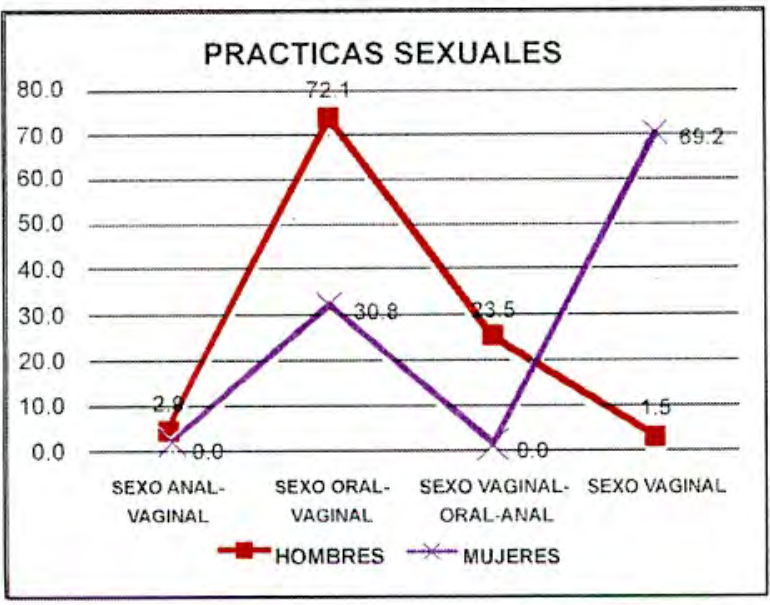

Figura $N^{\circ} 1$. Distribución de Frecuencias Según Prácticas Sexuales de los Estudiantes Universitarios, de la Universidad Nacional Jorge Basadre Grohmann Tacna-Peru 2009

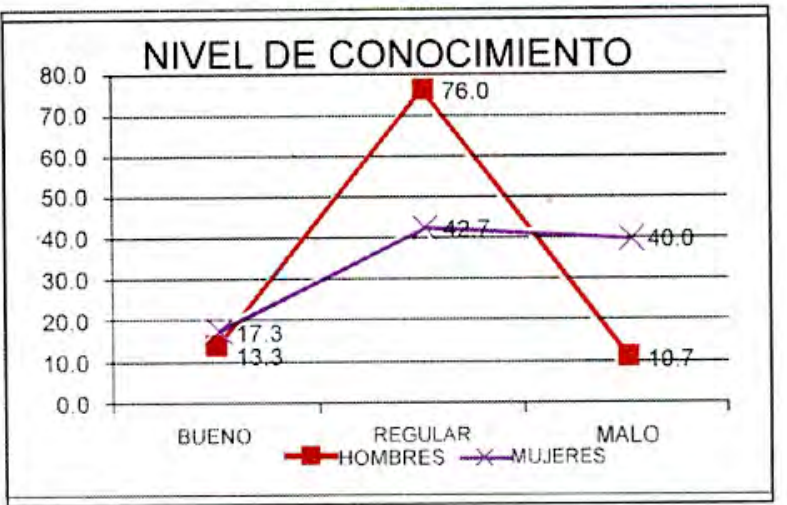

Figura $\mathrm{N}^{\circ}$ 02. Distribución de Frecuencias Según el Nivel de Conocimiento de los Estudiantes Universitarios, de la Universidad Nacional Jorge Basadre Grohmann Tacna-Peru 2009

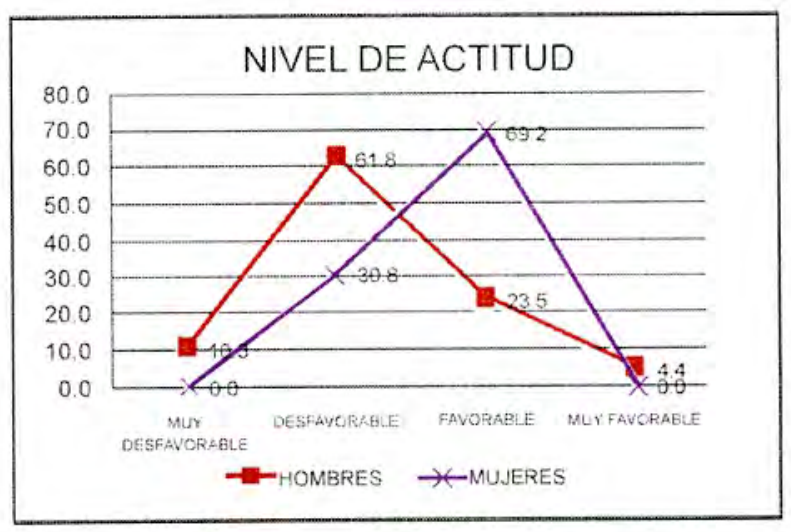

Figura 3. Distribución de Frecuencias según el nivel de actitud de los estudiantes universitarios, de la Universidad Nacional Jorge Basadre Grohmann, TacnaPerú 2009

\section{DISCUSIÓN}

Los resultados del presente estudio nos orienta a señalar que los jóvenes universitarios varones iniciaron su primera relación coital más tempranamente, entre 16 a 20 años a diferencia de las mujeres que lo hicieron entre los 21 a 25 años, lo cual no coincide con los resultados del estudio realizado por M.G. Lasheras Pérez, España: "Hábitos sexuales en jóvenes universitarios", en donde encontraron que las edades de inicio de las relaciones coitales para varones fue de 14 años y de 16 años para las mujeres. Lo que permite concluir que la mujer posterga el inicio de las relaciones coitales, lo cual muestra conducta orientada a mayor responsabilidad respecto a su sexualidad y frente a las ITS y VIH/SIDA.

El nivel de conocimiento sobre sexualidad y sexo en la población universitaria estudiada fue de regular a malo, lo que repercute en el comportamiento sexual y los expone a riesgos sexuales y reproductivos. Por otra parte la actitud mostrada para evitar contraer Infecciones de transmisión sexual por los varones es desfavorable, en relación a la actitud favorable presentada por las mujeres.

Es necesario posicionar la actitud y conducta responsable en materia de sexualidad, 
complementando esfuerzos con el sector educación y salud. Las autoridades de educación y salud deben intervenir más coordinadamente con programas intersectoriales preventivo promocionales, en relación con la salud sexual reproductiva al interior de la universidad.

Las autoridades universitarias, Centros federados y otras organizaciones deben coordinar planes y acciones para generar una sexualidad sana y medidas concretas que permitan reforzar los conocimientos sobre salud sexual y reproductiva.

\section{REFERENCIAS BIBLIOGRÁFICAS}

Botero, J. "Obstetricia y Ginecología", 7ma ed. Medellin Colombia; 2004

Debuse, M. Lo esencial en Sistema Endocrino y Aparato Reproductor. 2da ed. Harcourt Brace; 2005.

Donas S. Adolescencia y Juventud. San José, Costa Rica: OPS/OMS; 1997.

Erikson, E. Identidad, Juventud y Crisis. Buenos Aires: Editorial Raidos; 1974

González Merlo J. Obstetricia. 2da ed. Barcelona España: Salvat Editores; 1986

Ministerio de Salud. Guía de Bolsillo para los proveedores de servicios de Planificación Familiar. Lima. 1995.
Ministerio de Salud. Guías Nacionales de Atención Integral de la Salud Sexual y Reproductiva. Dirección General de Salud de las personas. Dirección de Programas Sociales Lima. 2004.

Munist, T., Maddaleno, M. y Suárez, E. Manual de Medicina de la Adolescencia. Serie PALTEX No. 20. Washington: OPS

Organización Panamericana de la Salud. Reforma del sector salud y derechos sexuales y reproductivos OPS/PALTEX. 2004.

Pérez Sánchez, A. Obstetricia. 3ra ed. Santiago Chile. Mediterráneo. 1999.

Silber, T. Prevención y Promoción de la Salud Adolescente. En Silben 1992

Suárez, E.N. y Krauskopf, D. El enfoque de Riesgo y su Aplicación a las Conductas en la Adolescencia. Una Perspectiva Psicosocial. Washington: OPS

Wetnstein, J. Riesgo Psicosocial en Jóvenes. Santiago de Chile: PREALC; 1992

\section{Correspondencia:}

Leandra Llanca Ramos

Ciudad Universitaria Fundo "Los Granados"

Av. Miraflores s/n. Tacna. Perú

leatacna@yahoo.es 